\title{
The USTC-NELSLIP Systems for Simultaneous Speech Translation Task at IWSLT 2021
}

\author{
Dan $\mathrm{Liu}^{1,2}$, Mengge $\mathrm{Du}^{2}$, Xiaoxi $\mathrm{Li}^{2}$, Yuchen $\mathrm{Hu}^{1}$, and Lirong Dai ${ }^{1}$ \\ ${ }^{1}$ University of Science and Technology of China, Hefei, China \\ ${ }^{2}$ iFlytek Research, Hefei, China \\ \{danliu, huyuchen\}@mail.ustc.edu.cn \\ lrdai@ustc.edu.cn \\ \{danliu,xxli16,mgdou\}@iflytek.com
}

\begin{abstract}
This paper describes USTC-NELSLIP's submissions to the IWSLT2021 Simultaneous Speech Translation task. We proposed a novel simultaneous translation model, Cross Attention Augmented Transducer (CAAT), which extends conventional RNN-T to sequence-tosequence tasks without monotonic constraints, e.g., simultaneous translation. Experiments on speech-to-text (S2T) and text-to-text (T2T) simultaneous translation tasks shows CAAT achieves better quality-latency trade-offs compared to wait-k, one of the previous state-ofthe-art approaches. Based on CAAT architecture and data augmentation, we build S2T and T2T simultaneous translation systems in this evaluation campaign. Compared to last year's optimal systems, our S2T simultaneous translation system improves by an average of 11.3 BLEU for all latency regimes, and our T2T simultaneous translation system improves by an average of 4.6 BLEU.
\end{abstract}

\section{Introduction}

This paper describes the submission to IWSLT 2021 Simultaneous Speech Translation task by National Engineering Laboratory for Speech and Language Information Processing (NELSLIP), University of Science and Technology of China, China.

Recent work in text-to-text simultaneous translation tends to fall into two categories, fixed policy and flexible policy, represented by wait-k (Ma et al., 2019) and monotonic attention (Arivazhagan et al., 2019; Ma et al., 2020b) respectively. The drawback of fixed policy is that it may introduce over latency for some sentences and under latency for others. Meanwhile, flexible policy often leads to difficulties in model optimization.

Inspired by RNN-T (Graves, 2012), we aim to optimize the marginal distribution of all expanded paths in simultaneous translation. However, we found it's impossible to calculate the marginal probability based on conventional Attention Encoder-Decoder (Sennrich et al., 2016) architectures (Transformer (Vaswani et al., 2017) included), which is due to the deep coupling between source contexts and target history contexts. To solve this problem, we propose a novel architecture, Cross Attention augmented Transducer (CAAT), and a latency loss function to ensure CAAT model works with an appropriate latency. In simultaneous translation, policy is integrated into translation model and learned jointly for CAAT model.

In this work, we build simultaneous translation systems for both text-to-text (T2T) and speechto-text S2T) task. We propose a novel architecture, Cross Attention Augmented Transducer (CAAT), which significantly outperforms wait-k (Ma et al., 2019) baseline in both text-to-text and speech-to-text simultaneous translation task. Besides, we adopt a variety of data augmentation methods, back-translation (Edunov et al., 2018), Self-training (Kim and Rush, 2016) and speech synthesis with Tacotron2 (Shen et al., 2018). Combining all of these and models ensembling, we achieved about 11.3 BLEU (in S2T task) and 4.6 BLEU (in T2T task) gains compared to the best performance last year.

\section{Data}

\subsection{Statistics and Preprocessing}

EN $\rightarrow$ DE Speech Corpora The speech datasets used in our experiments are shown in Table 1, where MuST-C, Europarl and CoVoST2 are speech translation specific (speech, transcription and translation included), and LibriSpeech, TED-LIUM3 are speech recognition specific (only speech and transcription). After augmented with speed and echo perturbation, we use Kaldi (Povey et al., 2011) to extract 80 dimensional log-mel filter bank features, computed with a $25 \mathrm{~ms}$ window size and a 
$10 \mathrm{~ms}$ window shift, and specAugment (Park et al., 2019) were performed during training phase.

\begin{tabular}{ccc}
\hline Corpus & Segments & Duration(h) \\
\hline MuST-C & $250.9 \mathrm{k}$ & 448 \\
Europarl & $69.5 \mathrm{k}$ & 155 \\
CoVoST2 & $854.4 \mathrm{k}$ & 1090 \\
LibriSpeech & $281.2 \mathrm{k}$ & 960 \\
TED-LIUM3 & $268.2 \mathrm{k}$ & 452 \\
\hline
\end{tabular}

Table 1: Statistics of speech corpora.

Text Translation Corpora The bilingual parallel datasets for Englith to German $(\mathrm{EN} \rightarrow \mathrm{DE})$ and English to Japanese $(\mathrm{EN} \rightarrow \mathrm{JA})$ used are shown in Table 2, and the monolingual datasets in English, German and Japanese are shown in Table 3. And we found the Paracrawl dataset in $\mathrm{EN} \rightarrow \mathrm{DE}$ task is too big to our model training, we randomly select a subset of $14 \mathrm{M}$ sentences from it.

\begin{tabular}{ccc}
\hline & Corpus & Sentences \\
\hline & MuST-C(v2) & $229.7 \mathrm{k}$ \\
& Europarl & $1828.5 \mathrm{k}$ \\
& Rapid-2019 & $1531.3 \mathrm{k}$ \\
$\mathbf{E N} \rightarrow \mathbf{D E}$ & WIT3-TED & $209.5 \mathrm{k}$ \\
& Commoncrawl & $2399.1 \mathrm{k}$ \\
& WikiMatrix & $6227.2 \mathrm{k}$ \\
& Wikititles & $1382.6 \mathrm{k}$ \\
& Paracrawl & $82638.2 \mathrm{k}$ \\
\hline \multirow{3}{*}{$\mathbf{E N} \rightarrow \mathbf{J A}$} & WIT3-TED & $225.0 \mathrm{k}$ \\
& JESC & $2797.4 \mathrm{k}$ \\
& kftt & $440.3 \mathrm{k}$ \\
& WikiMatrix & $3896.0 \mathrm{k}$ \\
& Wikititles & $706.0 \mathrm{k}$ \\
& Paracrawl & $10120.0 \mathrm{k}$ \\
\hline
\end{tabular}

Table 2: Statistics of text parallel datasets.

\begin{tabular}{ccc}
\hline Language & Corpus & Sentences \\
\hline \multirow{2}{*}{ EN } & Europarl-v10 & $2295.0 \mathrm{k}$ \\
& News-crawl-2019 & $33600.8 \mathrm{k}$ \\
\hline \multirow{2}{*}{ DE } & Europarl-v10 & $2108.0 \mathrm{k}$ \\
& News-crawl-2020 & $53674.4 \mathrm{k}$ \\
\hline \multirow{2}{*}{ JA } & News-crawl-2019 & $3446.4 \mathrm{k}$ \\
& News-crawl-2020 & $10943.3 \mathrm{k}$ \\
\hline
\end{tabular}

Table 3: Statistics of monolingual datasets.
For $\mathrm{EN} \rightarrow \mathrm{DE}$ task, we directly use SentencePiece (Kudo and Richardson, 2018) to generate a unigram vocabulary of size 32,000 for source and target language jointly. And for $\mathrm{EN} \rightarrow \mathrm{JA}$ task, sentences in Japanese are firstly participled by $\mathrm{MeCab}$ (Kudo, 2006), and then a unigram vocabulary of size 32,000 is generated for source and target jointly similar to $\mathrm{EN} \rightarrow \mathrm{DE}$ task.

During data preprocessing, the bilingual datasets are firstly filtered by length less than 1024 and length ratio of target to source $0.25<r<4$. In the second step, with a baseline Transformer model trained with only bilingual data, we filtered the mismatched parallel pairs with log-likelihood from the baseline model, threshold is set to -4.0 for $\mathrm{EN} \rightarrow \mathrm{DE}$ task and -5.0 for $\mathrm{EN} \rightarrow \mathrm{JA}$ task. At last we keep 27.3 million sentence pairs for EN-DE task and 17.0 sentence pairs for $\mathrm{EN} \rightarrow \mathrm{JA}$ task.

\subsection{Data Augmentation}

For text-to-text machine translation, augmented data from monolingual corpora in source and target language are generated by self-training ( $\mathrm{He}$ et al., 2019) and back translation (Edunov et al., 2018) respectively. Statistics of the augmented training data are shown in Table 4.

\begin{tabular}{lcc}
\hline Data & $\mathbf{E N} \rightarrow \mathbf{D E}$ & $\mathbf{E N} \rightarrow \mathbf{J A}$ \\
\hline Bilingual data & $27.3 \mathrm{M}$ & $17.0 \mathrm{M}$ \\
+back-translation & $34.3 \mathrm{M}$ & $22.0 \mathrm{M}$ \\
$\quad$ +self-training & $41.3 \mathrm{M}$ & $27.0 \mathrm{M}$ \\
\hline
\end{tabular}

Table 4: Augmented training data for text-to-text translation.

We further extend these two data augmentation methods to speech-to-text translation, detailed as:

1. Self-training: Maybe similar to sequencelevel distillation (Kim and Rush, 2016; Ren et al., 2020; Liu et al., 2019). Transcriptions of all speech datasets (both speech recognition and speech translation specific) are sent to a text translation model to generate text $y^{\prime}$ in target language, the generated $y^{\prime}$ with its corresponding speech are directly added to speech translation dataset.

2. Speech Synthesis: We employ Tacotron 2 (Shen et al., 2018) with slightly modified by introducing speaker representations to both encoder and decoder as our text-to-speech (TTS) model architecture, and trained on MuST$\mathrm{C}(\mathrm{v} 2)$ speech corpora to generate filter-bank 
speech representations. We randomly select $4 \mathrm{M}$ sentence pairs from $\mathrm{EN} \rightarrow \mathrm{DE}$ text translation corpora and generate audio feature by speech synthesis. The generated filter bank features and their corresponding target language text are used to expand our speech translation dataset.

The expanded training data are shown in Table 5. Besides, during the training period for all the speech translation tasks, we sample the speech data from the whole corpora with fixed ratio and the concrete ratio for different dataset is shown in $\mathrm{Ta}$ ble 6 .

\begin{tabular}{lcc}
\hline Dataset & Segements & Duration(h) \\
\hline Raw S2T dataset & $1.17 \mathrm{M}$ & 1693 \\
+self-training & $2.90 \mathrm{M}$ & 4799 \\
+Speech synthesis & $7.22 \mathrm{M}$ & 10424 \\
\hline
\end{tabular}

Table 5: Expanded speech translation dataset with selftraining and speech synthesis.

\begin{tabular}{cc}
\hline Dataset & Sample Ratio \\
\hline MuST-C & 2 \\
Europarl & 1 \\
CoVoST2 & 1 \\
LibriSpeech & 1 \\
TED-LIUM3 & 2 \\
Speech synthesis & 5 \\
\hline
\end{tabular}

Table 6: Sample ratio for different datasets during training period.

\section{Methods and Models}

\subsection{Cross Attention Augmented Transducer}

Let $\mathbf{x}$ and $\mathbf{y}$ denote the source and target sequence, respectively. The policy of simultaneous translation is denoted as an action sequence $\mathbf{p} \in\{R, W\}^{|\mathbf{x}|+|\mathbf{y}|}$ where $R$ denotes the READ action and $W$ the WRITE action. Another representation of policy is extending target sequence $\mathbf{y}$ to length $|\mathbf{x}|+|\mathbf{y}|$ with blank symbol $\phi$ as $\hat{y} \in(\mathbf{v} \cup\{\phi\})^{|\mathbf{x}|+|\mathbf{y}|}$, where $\mathbf{v}$ is the vocabulary of the target language. The mapping from $\mathbf{y}$ to sets of all possible expansion $\hat{y}$ denotes as $H(\mathbf{x}, \mathbf{y})$.

Inspired by RNN-T (Graves, 2012), the loss function for simultaneous translation can be defined as the marginal conditional probability and expecta- tion of latency metric through all possible expanded paths:

$$
\begin{aligned}
& \mathcal{L}(x, y)=\mathcal{L}_{\text {nll }}(x, y)+\mathcal{L}_{\text {latency }}(x, y) \\
& =-\log \sum_{\hat{y}} p(\hat{y} \mid x)+\mathbb{E}_{\hat{y}} l(\hat{y}) \\
& =-\log \sum_{\hat{y}} p(\hat{y} \mid x)+\sum_{\hat{y}} \operatorname{Pr}(\hat{y} \mid y, x) l(\hat{y})
\end{aligned}
$$

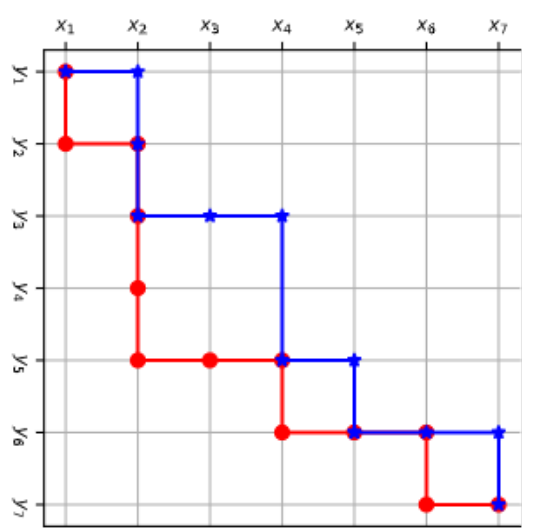

Figure 1: Expanded paths in simultaneous translation.

$$
\text { Where } \operatorname{Pr}(\hat{y} \mid y, x)=\frac{p(\hat{y} \mid x)}{\sum_{\hat{y}^{\prime} \in H(x, y)} p\left(\hat{y}^{\prime} \mid x\right)} \text {, and } \hat{y} \in
$$
$H(x, y)$ is an expansion of target sequence $\mathbf{y}$, and $l(\hat{y}$ is the latency of expanded path $\hat{y}$.

However, RNN-T is trained and inferenced based on source-target monotonic constraint, which means it isn't suitable for translation task. And the calculation of marginal probability $\sum_{\hat{y} \in H(x, y)} \operatorname{Pr}(\hat{y} \mid x)$ is impossible for Attention Encoder-Decoder framework due to deep coupling of source and previous target representation. As shown in Figure 1, the decoder hidden states for the red path $\hat{y}^{1}$ and the blue path $\hat{y}^{2}$ is not equal at the intersection $s_{2}^{1} \neq s_{2}^{2}$. To solve this, we separate the source attention mechanism from the target history representation, which is similar to joiner and predictor in RNN-T. The novel architecture can be viewed as a extension version of RNN-T with attention mechanism augmented joiner, and is named as Cross Attention Augmented Transducer (CAAT). Figure 2 is the implementation of RAAT based on Transformer.

Computation cost of joiner in CAAT is significantly more expensive than that of RNN-T. The complexity of joiner is $\mathcal{O}(|\mathbf{x}| \cdot|\mathbf{y}|)$ during training, which means $\mathcal{O}(|\mathbf{x}|)$ times higher than conventional Transformer. We solve this problem by making decisions with decision step size $d>1$, and 


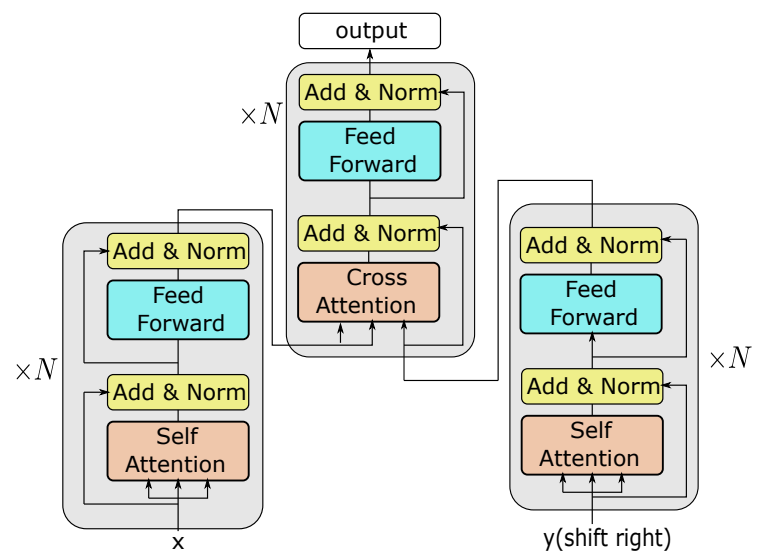

Figure 2: Architecture of CAAT based on Transformer.

reduce the complexity of joiner from $\mathcal{O}(|\mathbf{x}| \cdot|\mathbf{y}|)$ to $\frac{\mathcal{O}(|\mathbf{x}| \cdot|\mathbf{y}|)}{d}$. Besides, to further reduce video memory consumption, we split hidden states into small pieces before sent into joiner, and recombine it for back-propagation during training.

As the latency loss is defined as marginal expectation over all expanded paths $\hat{y}$, mergeable is also a requirement to the latency loss definition, which means latency loss through path $\hat{y}$ may be defined as $l(\hat{y})=\sum_{k=1}^{|\mathbf{x}|+|\mathbf{y}|} l\left(\hat{y}_{k}\right)$ and $l\left(\hat{y}_{k}\right)$ is independent of $\hat{y}_{j^{\prime} \neq j}$. However, both Average Lagging (Ma et al., 2019) and Differentiable Average Lagging (Arivazhagan et al., 2019) do not meet this requirement. We hence introduce a novel latency function based on wait- 0 as oracle latency as follows:

$$
\begin{array}{r}
d(i, j)=\frac{1}{|\mathbf{y}|} \max \left(i-\frac{j \cdot|\mathbf{x}|}{|\mathbf{y}|}, 0\right) \\
l\left(\hat{y}_{k}\right)= \begin{cases}0 & \text { if } \hat{y}_{k}=\phi \\
d\left(i_{k}, j_{k}\right) & \text { else }\end{cases}
\end{array}
$$

Where $i_{k}=\sum_{k^{\prime}=1}^{k} I\left(\hat{y}_{k^{\prime}}=\phi\right)$ and $j_{k}=$ $\sum_{k^{\prime}=1}^{k} I\left(\hat{y}_{k^{\prime}} \neq \phi\right)$ denote READ and WRITE actions number before $\hat{y}_{k}$. The latency for the whole expanded path $\hat{y}$ can be defined as

$$
l(\hat{y})=\sum_{k=1}^{|\hat{\mathbf{y}}|} l\left(\hat{y}_{k}\right)
$$

Based on Eq. (3) the expectation of latency loss through all expanded paths may be defined as :

$$
\begin{aligned}
\mathcal{L}_{\text {latency }}(x, y) & =\mathbb{E}_{\hat{y} \in H(x, y)} l(\hat{y}) \\
& =\sum_{\hat{y}} \operatorname{Pr}(\hat{y} \mid y, x) l(\hat{y})
\end{aligned}
$$

Latency loss and its gradients can be calculated by the forward-backward algorithm, similar to Sequence Criterion Training in ASR (Povey, 2005).

At last, we add the cross entropy loss of offline translation model as an auxiliary loss to CAAT model training for two reasons. First we hope the CAAT model fall back to offline translation in the worst case; second, CAAT models is carried out in accordance with offline translation when source sentence ended. The final loss function for CAAT training is defined as follows:

$$
\begin{aligned}
\mathcal{L}(x, y) & =\mathcal{L}_{C A A T}(x, y)+\lambda_{\text {latency }} \mathcal{L}_{\text {latency }}(x, y) \\
& +\lambda_{C E} \mathcal{L}_{C E}(x, y) \\
& =-\log \sum_{\hat{y}} p(\hat{y} \mid x) \\
& +\lambda_{\text {latency }} \sum_{\hat{y}} \operatorname{Pr}(\hat{y} \mid y, x) d(\hat{y}) \\
& -\lambda_{C E} \sum_{j} \log p\left(y_{j} \mid x, y_{<j}\right)
\end{aligned}
$$

Where $\lambda_{\text {latency }}$ and $\lambda_{C E}$ are scaling factors corresponding to the $\mathcal{L}_{\text {latency }}$ and $\mathcal{L}_{C E}$. And we set $\lambda_{1}=\lambda_{2}=1.0$ if not specified.

\subsection{Streaming Encoder}

Unidirectional Transformer encoder (Arivazhagan et al., 2019; Ma et al., 2020b) is not effective for speech data processing, because of the closely related to right context for speech frame $x_{i}$. Block processing (Dong et al., 2019; Wu et al., 2020) is introduced for online ASR, but they lacks directly observing to infinite left context.

We process streaming encoder for speech data by block processing with right context and infinite left context. First, input representations $\mathbf{h}$ is divided into overlapped blocks with block step $m$ and block size $m+r$. Each block consists of two parts, the main context $\mathbf{m}_{n}=$ $\left[h_{m * n+1}, \cdots, h_{(m+1) * n}\right]$ and the right context $\mathbf{r}_{n}=\left[h_{(m+1) * n}, \cdots, h_{(m+1) * n+r}\right]$. The query, key and value of block $\mathbf{b}_{n}$ in self-attention can be described as follows:

$$
\begin{aligned}
& \mathbf{Q}=\mathbf{W}_{q}\left[\mathbf{m}_{n}, \mathbf{r}_{n}\right] \\
& \mathbf{K}=\mathbf{W}_{k}\left[\mathbf{m}_{1}, \cdots, \mathbf{m}_{n}, \mathbf{r}_{n}\right] \\
& \mathbf{V}=\mathbf{W}_{v}\left[\mathbf{m}_{1}, \cdots, \mathbf{m}_{n}, \mathbf{r}_{n}\right]
\end{aligned}
$$

By reorganizing input sequence and designed self-attention mask, training is effective by reusing conventional transformer encoder layers. And unidirectional transformer can be regarded as a special 
case of our method with $\{m=1, r=0\}$. Note that the look-ahead window size in our method is fixed, which ensures increasing transformer layers won't affect latency.

\subsection{Text-to-Text Simultaneous Translation}

We implemented both CAAT in Sec. 3.1 and wait-k (Ma et al., 2019) systems for text-to-text simultaneous translation, both of them are implemented based on fairseq (Ott et al., 2019).

All of wait-k experiments use the parameter settings based on big transformer (Vaswani et al., 2017) with unidirectional encoders, which corresponds to a 12-layer encoder and 6-layer decoder transformer with a embedding size of 1024, a feed forward network size of 4096, and 16 heads attention.

Hyper-parameters of our CAAT model architectures are shown in Table 7. CAAT training requires significantly more GPU memory than conventional Transformer (Vaswani et al., 2017), for the $\mathcal{O}\left(\frac{|x| \cdot|y|}{d}\right)$ complexity of joiner module. We mitigate this problem by reducing joiner hidden dimension for lower decision step size $d$.

\subsection{Speech-to-Text Simultaneous Translation}

\subsubsection{End-to-End Systems}

The main system of End-to-End Speech-to-Text simultaneous Translation is based on the aforementioned CAAT structure. For speech encoder, two 2D convolution blocks are introduced before the stacked 24 Transformer encoder layers. Each convolution block consists of a 3-by-3 convolution layer with 64 channels and stride size as 2 , and a ReLU activation function. Input speech features are downsampled 4 times by convolution blocks and flattened to 1D sequence as input to transformer layers. Other hyper-parameters are shown in Table 7. The latency-quality trade-off may be adjusted by varying the decision step size $d$ and the latency scaling factor $\lambda_{\text {latency }}$. We submitted systems with best performance in each latency region.

\subsubsection{Cascaded Systems}

The cascaded system consists of two modules, simultaneous automatic speech recognition (ASR) and simultaneous text-to-text Machine Translation (MT). Both simultaneous ASR and MT system are built with CAAT proposed in Sec. 3.1. And we found the cascaded systems outperforms end-toend system in medium and high latency region.

\subsection{Unsegmented Data Processing}

To deal with unsegmented data, we segment the input text based on sentence ending marks for T2T track. For S2T task, input speech is simply segmented into utterances with duration of $20 \mathrm{sec}$ onds and each segmented piece is directly sent to our simultaneous translation systems to obtain the streaming results. We found an abnormally large average lagging $(A L)$ on IWSLT tst2018 test set based on existed SimuEval toolkit(Ma et al., 2020a) and segment strategy, so relevant results are not presented here. A more reasonable latency criterion may be needed for unsegmented data in the future.

\section{Experiments}

\subsection{Effectiveness of CAAT}

To demonstrate the effectiveness of CAAT architecture, we compare it to wait-k with speculative beam search (SBS) (Ma et al., 2019; Zheng et al., 2019b), one of the previous state-of-the-art. The latencyquality trade-off curves on S2T and T2T tasks are shown in Figure 3 and Figure 4(a). We can find that CAAT significantly outperforms wait-k with SBS, especially in low latency $\operatorname{section}(A L<1000 \mathrm{~ms}$ for $\mathrm{S} 2 \mathrm{~T}$ track and $A L<3$ for T2T track).

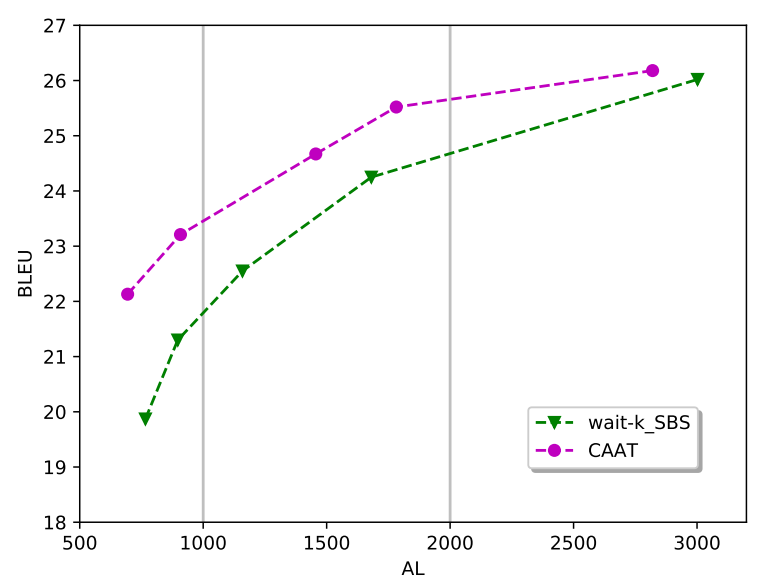

Figure 3: Comparison of CAAT and wait-k with $\mathrm{SBS}$ systems on $\mathrm{EN} \rightarrow \mathrm{DE}$ Speech-to-Text simultaneous translation.

\subsection{Effectiveness of data augmentation}

In order to testify the effectiveness of data augmentation, we compare the results of different data augmentation methods based on the offline and simultaneous speech translation task. As demonstrated in Table 8 , adding new generated target sentences into the training corpora by using Self-training gives 


\begin{tabular}{ccccc}
\hline \multirow{4}{*}{ Encoder } & Parameters & S2T config & T2T config-A & T2T config-B \\
\hline \multirow{5}{*}{ Predictor } & layers & 24 & 12 & 12 \\
& attention heads & 8 & 16 & 16 \\
& FFN dimension & 2048 & 4096 & 4096 \\
& embedding size & 512 & 1024 & 1024 \\
\hline \multirow{3}{*}{ Joiner } & attention heads & 8 & 16 & 16 \\
& FFN dimension & 2048 & 4096 & 4096 \\
& embedding size & 512 & 1024 & 1024 \\
& output dimension & 512 & 512 & 1024 \\
\hline \multirow{2}{*}{$/$} & attention heads & 8 & 8 & 16 \\
& FFN dimension & 1024 & 2048 & 4096 \\
& embedding size & 512 & 512 & 1024 \\
\hline
\end{tabular}

Table 7: Parameters of CAAT in T2T and end-to-end S2T simultaneous translation. Noted that both predictor and joiner have 6 layers for T2T and S2T tasks, and the additional two parameters for end-to-end 2T simultaneous translation, which is the main context and right context described in Sec.3.2, are set $m=32$ and $r=16$.

\begin{tabular}{lc}
\hline Dataset & BLEU \\
\hline Original speech corpora & 21.24 \\
+self-training & 28.21 \\
+Speech systhesis & 29.72 \\
\hline
\end{tabular}

Table 8: Performance of offline speech translation on MuST-C(v2) tst-COMMON with different datasets.

a boost of nearly 7 BLEU points and speech synthesis provides the other 1.5 BLEU points increase on MuST-C(v2) tst-COMMON. As illustrated in Figure 3, all the data augmentation methods are employed and provide nearly 3 BLEU points on average in the simultaneous task at different latency regimes. Note that our data augmentation methods alleviate the scarcity of parallel datasets in the End-to-End speech translation task and make a significant improvement.

\subsection{Text-to-Text Simultaneous Translation}

$\mathbf{E N} \rightarrow \mathbf{D E}$ Task The performances of text-to-text $\mathrm{EN} \rightarrow \mathrm{DE}$ task is shown in Figure 4(a). We can see that the performance of proposed CAAT is always better than that of wait-k with SBS and the best results from ON-TRAC in 2020 (Elbayad et al., 2020), especially in low latency regime, and the performance of CAAT with model ensemble is nearly equivalent to offline result. Moreover, it can be further noticed from Figure 4(a) that the model ensemble can also improve the BLUE score more or less under different latency regimes, and the increase is quite obvious in low latency regime. Compared with the best result in 2020, we finally get improvement by 6.8 and 3.4 BLEU in low and high latency regime respectively.

$\mathbf{E n} \rightarrow \mathbf{J A}$ Task Results of Text-to-Text simultaneous translation $(\mathrm{EN} \rightarrow \mathrm{JA})$ track are plotted in Figure 4(b), where the curve naming CAAT_bst is best performances in this track with or without modelensembling method. Curves in this sub-figure show the similar conclusion to the former subsection, that the result of proposed CAAT significantly outperforms that of wait-k with SBS. While we can also find that the gap between CAAT and offline is more obvious (nearly 0.4 BLEU), this is mainly because parameters of joiner block for EN $\rightarrow \mathrm{JA}$ track in high-latency regime is reduced a lot from that for $\mathrm{EN} \rightarrow \mathrm{DE}$ track, due to the unstable $\mathrm{EN} \rightarrow \mathrm{JA}$ training.

\subsection{Speech-to-Text Simultaneous Translation}

End-to-End System In this section, we discuss about our final results of End-to-End system based on CAAT. We tune the decision step size $d$ and latency scaling factor $\lambda_{\text {latency }}$ to meet different latency regime requirements. For low, medium and high latency, the corresponding $d$ and $\lambda_{\text {latency }}$ are set to $(16,64,64)$ and $(1.0,1.0,0.2)$ respectively. We show our final latency-quality trade-offs in Figure 5. Combined with our data augmentation methods and 


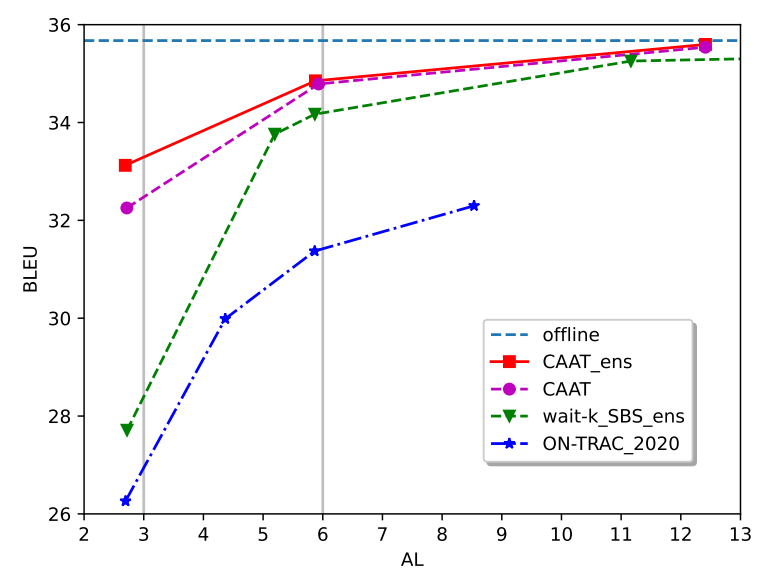

(a) tst-COMMON(v2) on $\mathrm{EN} \rightarrow \mathrm{DE}$

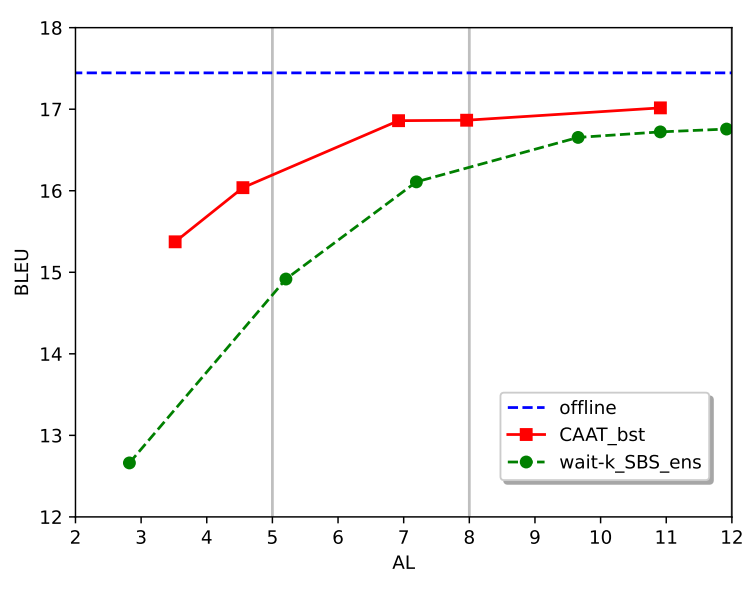

(b) IWSLT dev2021 on EN $\rightarrow$ JA

Figure 4: Latency-quality trade-offs of Text-to-Text simultaneous translation.

new CAAT model structure, it can be seen that our single model system has already outperformed the best results of last year in all latency regimes and provides 9.8 BLEU scores increase on average. Ensembling different models can further boost the BLEU scores by roughly $0.5-1.5$ points at different latency regimes.

Cascaded System Under the cascaded setting, we paired two well-trained ASR and MT systems, where the WER of ASR system's performance is 6.30 with $1720.20 \mathrm{AL}$, and the MT system is followed by the config-A in Table 7, whose results are 34.79 BLEU and 5.93 AL. We found the best medium and high-latency systems at decision step size pair $\left(d_{a s r}, d_{m t}\right)$ with $(6,10)$ and $(12,10)$ respectively. Performance of cascaded systems are shown in Figure 5. Note that under current configuration of ASR and MT systems, we can not provide valid results that satisfy the requirement of $A L$ at low latency regime since cascaded system usually has a larger latency compared to End-to End system. During the online decoding of the cascaded system, only after specific tokens are recognized by the ASR system, the translation model can further translate them to obtain the final result. The decoded results from ASR model first has a delay compared to the actual contents of the audio, and the two-steps decoding further accumulates the delay, which contributes to the higher latency compared to the End-to-End system. However, it still can be seen that cascaded system has significant advantages over End-to-End system at medium and high latency regime and it still has a long way to go for End-to-End system in the simultaneous speech translation task.

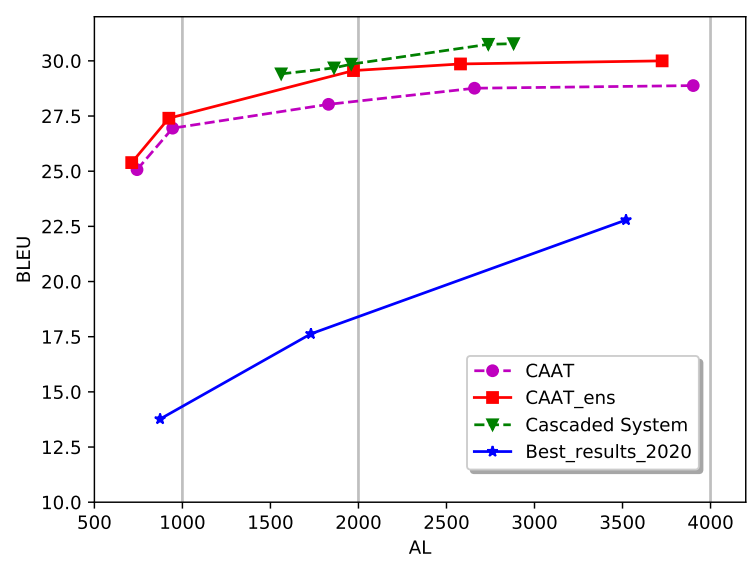

Figure 5: Latency-quality trade-offs of Speech-toText simultaneous translation on MuST-C(v2) tstCOMMON.

\section{Related Work}

Simultaneous Translation Recent work on simultaneous translation falls into two categories. The first category uses a fixed policy for the READ/WRITE actions and can thus be easily integrated into the training stage, as typified by wait$k$ approaches (Ma et al., 2019).The second category includes models with a flexible policy learned and/or adaptive to current context, e.g., by Reinforcement Learning (Gu et al., 2017), Supervise Learning (Zheng et al., 2019a) and so on. A special sub-category of flexible policy jointly optimizes policy and translation by monotonic attention customized to translation model, e.g., Monotonic Infinite Lookback (MILk) attention (Arivazhagan et al., 
2019) and Monotonic Multihead Attention (MMA) (Ma et al., 2020b). We propose a novel method to optimize policy and translation model jointly, which is motivated by RNN-T (Graves, 2012) in online ASR. Unlike RNN-T, the CAAT model removes the monotonic constraint, which is critical for considering reordering in machine translation tasks. The optimization of our latency loss is motivated by Sequence Discriminative Training in ASR (Povey, 2005).

Data Augmentation As described in Sec. 2, the size of training data for speech translation is significantly smaller than that of text-to-text machine translation, which is the main bottleneck to improve the performance of speech translation. Selftraining, or sequnece-level knowledge distillation by text-to-text machine translation model, is the most effective way to utilize the huge ASR training data (Liu et al., 2019; Pino et al., 2020). On the other hand, synthesizing data by text-to-speech (TTS) has been demonstrated to be effective for low resource speech recognition task (Gokay and Yalcin, 2019; Ren et al., 2019). To the best of our knowledge, this is the first work to augment data by TTS for simultaneous speech-to-text translation tasks.

\section{Conclusion}

In this paper, we propose a novel simultaneous translation architecture, Cross Attention Augmented Transducer (CAAT), which significantly outperforms wait-k in both S2T and T2T simultaneous translation task. Based on CAAT architecture and data augmentation, we build simultaneous translation systems on text-to-text and speech-totext simultaneous translation tasks. We also build a cascaded speech-to-text simultaneous translation system for comparison. Both T2T and S2T systems achieve significant improvements over last year's best-performing systems.

\section{References}

Naveen Arivazhagan, Colin Cherry, Wolfgang Macherey, Chung-Cheng Chiu, Semih Yavuz, Ruoming Pang, Wei Li, and Colin Raffel. 2019. Monotonic infinite lookback attention for simultaneous machine translation. In Proceedings of the 57th Annual Meeting of the Association for Computational Linguistics, pages 1313-1323, Florence, Italy. Association for Computational Linguistics.
Linhao Dong, Feng Wang, and Bo Xu. 2019. Selfattention aligner: A latency-control end-to-end model for ASR using self-attention network and chunk-hopping. In IEEE International Conference on Acoustics, Speech and Signal Processing, ICASSP 2019, Brighton, United Kingdom, May 1217, 2019, pages 5656-5660. IEEE.

Sergey Edunov, Myle Ott, Michael Auli, and David Grangier. 2018. Understanding back-translation at scale. arXiv preprint arXiv:1808.09381.

Maha Elbayad, Ha Nguyen, Fethi Bougares, Natalia Tomashenko, Antoine Caubrière, Benjamin Lecouteux, Yannick Estève, and Laurent Besacier. 2020. On-trac consortium for end-to-end and simultaneous speech translation challenge tasks at iwslt 2020. arXiv preprint arXiv:2005.11861.

Ramazan Gokay and Hulya Yalcin. 2019. Improving low resource turkish speech recognition with data augmentation and tts. In 2019 16th International Multi-Conference on Systems, Signals \& Devices (SSD), pages 357-360. IEEE.

Alex Graves. 2012. Sequence transduction with recurrent neural networks. arXiv preprint arXiv:1211.3711.

Jiatao Gu, Graham Neubig, Kyunghyun Cho, and Victor O.K. Li. 2017. Learning to translate in real-time with neural machine translation. In Proceedings of the 15th Conference of the European Chapter of the Association for Computational Linguistics: Volume 1, Long Papers, pages 1053-1062, Valencia, Spain. Association for Computational Linguistics.

Junxian He, Jiatao Gu, Jiajun Shen, and Marc'Aurelio Ranzato. 2019. Revisiting self-training for neural sequence generation. arXiv preprint arXiv:1909.13788.

Yoon Kim and Alexander M Rush. 2016. Sequencelevel knowledge distillation. arXiv preprint arXiv:1606.07947.

Taku Kudo. 2006. Mecab: Yet another part-of-speech and morphological analyzer. http://mecab.sourceforge.jp.

Taku Kudo and John Richardson. 2018. SentencePiece: A simple and language independent subword tokenizer and detokenizer for neural text processing. In Proceedings of the 2018 Conference on Empirical Methods in Natural Language Processing: System Demonstrations, pages 66-71, Brussels, Belgium. Association for Computational Linguistics.

Yuchen Liu, Hao Xiong, Zhongjun He, Jiajun Zhang, Hua Wu, Haifeng Wang, and Chengqing Zong. 2019. End-to-end speech translation with knowledge distillation.

Mingbo Ma, Liang Huang, Hao Xiong, Renjie Zheng, Kaibo Liu, Baigong Zheng, Chuanqiang Zhang, Zhongjun He, Hairong Liu, Xing Li, Hua Wu, and 
Haifeng Wang. 2019. STACL: Simultaneous translation with implicit anticipation and controllable latency using prefix-to-prefix framework. In Proceedings of the 57th Annual Meeting of the Association for Computational Linguistics, pages 3025-3036, Florence, Italy. Association for Computational Linguistics.

Xutai Ma, Mohammad Javad Dousti, Changhan Wang, Jiatao Gu, and Juan Pino. 2020a. SIMULEVAL: An evaluation toolkit for simultaneous translation. In Proceedings of the 2020 Conference on Empirical Methods in Natural Language Processing: System Demonstrations, pages 144-150, Online. Association for Computational Linguistics.

Xutai Ma, Juan Miguel Pino, James Cross, Liezl Puzon, and Jiatao Gu. 2020b. Monotonic multihead attention. In 8th International Conference on Learning Representations, ICLR 2020, Addis Ababa, Ethiopia, April 26-30, 2020. OpenReview.net.

Myle Ott, Sergey Edunov, Alexei Baevski, Angela Fan, Sam Gross, Nathan Ng, David Grangier, and Michael Auli. 2019. fairseq: A fast, extensible toolkit for sequence modeling. In Proceedings of the 2019 Conference of the North American Chapter of the Association for Computational Linguistics (Demonstrations), pages 48-53, Minneapolis, Minnesota. Association for Computational Linguistics.

Daniel S Park, William Chan, Yu Zhang, Chung-Cheng Chiu, Barret Zoph, Ekin D Cubuk, and Quoc V Le. 2019. Specaugment: A simple data augmentation method for automatic speech recognition. arXiv preprint arXiv:1904.08779.

Juan Pino, Qiantong Xu, Xutai Ma, Mohammad Javad Dousti, and Yun Tang. 2020. Self-training for end-to-end speech translation. arXiv preprint arXiv:2006.02490.

Daniel Povey. 2005. Discriminative training for large vocabulary speech recognition. Ph.D. thesis, University of Cambridge.

Daniel Povey, Arnab Ghoshal, Gilles Boulianne, Lukas Burget, Ondrej Glembek, Nagendra Goel, Mirko Hannemann, Petr Motlicek, Yanmin Qian, Petr Schwarz, et al. 2011. The kaldi speech recognition toolkit. In IEEE 2011 workshop on automatic speech recognition and understanding, CONF. IEEE Signal Processing Society.

Yi Ren, Jinglin Liu, Xu Tan, Chen Zhang, Tao Qin, Zhou Zhao, and Tie-Yan Liu. 2020. SimulSpeech: End-to-end simultaneous speech to text translation. In Proceedings of the 58th Annual Meeting of the Association for Computational Linguistics, pages 3787-3796, Online. Association for Computational Linguistics.

Yi Ren, Xu Tan, Tao Qin, Sheng Zhao, Zhou Zhao, and Tie-Yan Liu. 2019. Almost unsupervised text to speech and automatic speech recognition. In International Conference on Machine Learning, pages 5410-5419. PMLR.

Rico Sennrich, Barry Haddow, and Alexandra Birch. 2016. Neural machine translation of rare words with subword units. In Proceedings of the 54th Annual Meeting of the Association for Computational Linguistics (Volume 1: Long Papers), pages 17151725, Berlin, Germany. Association for Computational Linguistics.

Jonathan Shen, Ruoming Pang, Ron J Weiss, Mike Schuster, Navdeep Jaitly, Zongheng Yang, Zhifeng Chen, Yu Zhang, Yuxuan Wang, Rj Skerrv-Ryan, et al. 2018. Natural tts synthesis by conditioning wavenet on mel spectrogram predictions. In 2018 IEEE International Conference on Acoustics, Speech and Signal Processing (ICASSP), pages 4779-4783. IEEE.

Ashish Vaswani, Noam Shazeer, Niki Parmar, Jakob Uszkoreit, Llion Jones, Aidan N. Gomez, Lukasz Kaiser, and Illia Polosukhin. 2017. Attention is all you need. In Advances in Neural Information Processing Systems 30: Annual Conference on Neural Information Processing Systems 2017, December 49, 2017, Long Beach, CA, USA, pages 5998-6008.

Chunyang Wu, Yongqiang Wang, Yangyang Shi, Ching-Feng Yeh, and Frank Zhang. 2020. Streaming transformer-based acoustic models using selfattention with augmented memory. arXiv preprint arXiv:2005.08042.

Baigong Zheng, Renjie Zheng, Mingbo Ma, and Liang Huang. 2019a. Simultaneous translation with flexible policy via restricted imitation learning. In Proceedings of the 57th Annual Meeting of the Association for Computational Linguistics, pages 58165822, Florence, Italy. Association for Computational Linguistics.

Renjie Zheng, Mingbo Ma, Baigong Zheng, and Liang Huang. 2019b. Speculative beam search for simultaneous translation. In Proceedings of the 2019 Conference on Empirical Methods in Natural Language Processing and the 9th International Joint Conference on Natural Language Processing (EMNLPIJCNLP), pages 1395-1402, Hong Kong, China. Association for Computational Linguistics. 